\title{
Effects of Shenfu Injection (参附注射液) on Inflammatory Response during Post-Resuscitation Myocardial Dysfunction after Cardiac Arrest in Swine*
}

\author{
GU Wei ${ }^{1 \triangle}$, HOU Xiao-min ${ }^{2}$, and LI Chun-sheng ${ }^{2}$
}

\begin{abstract}
Objective: To investigate whether Shenfu Injection (SFI, 参附注射液) can alleviate post-resuscitation myocardial dysfunction by inhibiting the inflammatory response. Methods: After 8 min of ventricular fibrillation and $2 \mathrm{~min}$ of basic life support, 24 pigs were randomly divided into 3 groups ( $n=8$ ), which were given intravenous bolus injections of SFI $(1.0 \mathrm{~mL} / \mathrm{kg})$, epinephrine $(E P, 0.02 \mathrm{mg} / \mathrm{kg})$ and normal saline (SA), respectively. The animals were sacrificed at $24 \mathrm{~h}$ after restoration of spontaneous circulation (ROSC), and serum interleuking-6 (IL-6) and tumor necrosis factor- $\alpha$ (TNF- $\alpha$ ) levels were measured by enzyme-linked immunosorbent assay (ELISA); expressions of Toll-like receptor 4 (TLR4)/nuclear factor kappa B (NF- $\kappa$ B) mRNAs and proteins were determined by RT-PCR and Western blot, respectively. Results: Compared with the EP and the SA groups, the ultrastructure of myocardial cells were slightly damaged and the systolic function of the left ventricle was markedly improved in the SFI group at $24 \mathrm{~h}$ after ROSC $(P<0.05)$. In addition, compared with the EP and SA groups, the SFI group also showed significantly reduced levels of serum IL- 6 and TNF- $\alpha$, protein and mRNA levels of myocardial NF- $\kappa$ B and TLR4 $(P<0.05)$. Conclusions: Activation of TLR4/NF- $\kappa$ B signaling pathway may be involved in the pathological mechanisms of post-resuscitation myocardial dysfunction. SFI may block NF- $\kappa$ B-mediated inflammatory response by reducing the activity of NF- $\kappa$ B and the level of TNF- $\alpha$, thus playing a protective role in post-resuscitation myocardial dysfunction.
\end{abstract}

KEYWORDS Shenfu Injection, Chinese medicine, cardiopulmonary resuscitation, post-resuscitation myocardial dysfunction, TLR4/NF- $\kappa$ B

Although tremendous efforts have been made in scientific research to improve the survival rate of cardiac arrest (CA), and restoration of spontaneous circulation (ROSC) is achieved after cardiopulmonary resuscitation (CPR) in $20 \%$ to $40 \%$ of patients, only $1.4 \%$ to $17 \%$ of patients are discharged alive and post-resuscitation myocardial dysfunction occurs at $72 \mathrm{~h}$ after ROSC in most of patients. ${ }^{(1)}$ The main mechanism involves myocardial ischemia-reperfusion injury, ${ }^{(2)}$ cell apoptosis, ${ }^{(3)}$ calcium regulation disorder and immune dysfunction. ${ }^{(4,5)}$ During CA and CPR, the body experiences strong stress responses and produces a large amount of cytokines and inflammatory mediators, including tumor necrosis factor- $\alpha$ (TNF- $\alpha$ ) and interleukin-6 (IL-6), to directly act upon the myocardial cells and impair heart function. ${ }^{(5)}$ Tolllike receptor 4 (TLR4), the most common member of the Toll-like receptor family, is the endogenous receptor of lipopolysaccharide (LPS). TLR4 can activate the downstream inflammatory pathways after LPS stimulation, leading to release of inflammatory mediators including TNF- $\alpha$ and IL-6, and consequent injury, necrosis and apoptosis of myocardial cells. ${ }^{(6)}$

Shenfu Injection (SFI, 参附注射液) is a famous traditional Chinese medicine preparation and the main components are ginsenoside and aconitine, which can stimulate myocardium, increase the contractility of myocardium, and enhance the blood flow velocity

CThe Chinese Journal of Integrated Traditional and Western Medicine Press and Springer-Verlag GmbH Germany, part of Springer Nature 2021

*Supported by the Beijing Natural Science Foundation (No. 7182055) and Beijing Municipal Administration of Hospitals Incubating Program (No. 2016022)

1. Department of Emergency Medicine, Beijing Friendship Hospital, Capital Medical University, Beijing Key Laboratory of Cardiopulmonary Cerebral Resuscitation, Beijing (100050), China; 2. Department of Emergency Medicine, Beijing Chaoyang Hospital, Capital Medical University, Beijing Key Laboratory of Cardiopulmonary Cerebral Resuscitation, Beijing (100020), China Correspondence to: Prof. LI Chun-sheng, E-mail: Icscyyy @ 163.com ${ }^{\triangle}$ Now the author is at Emergency Medicine Center, Chui Yang Liu Hospital Affiliated to Tsinghua University, Beijing (100022), China DOI: https://doi.org/10.1007/s11655-021-2855-2 
of the coronary artery. Our previous animal studies have confirmed that SFI significantly improved tissue perfusion and oxygen metabolism, alleviated ischemia reperfusion, increased cardiac output, and inhibited the apoptosis of myocardial cells, playing a protective role in post-resuscitation myocardial dysfunction. ${ }^{(7,8)}$ Whether SFI exerts such protective function by modulating the TLR4/nuclear factor kappa B (NF- $\kappa$ B) signaling pathway has not yet been demonstrated. Therefore, the present study established the 8-min CA model of miniature pigs and observe protein and mRNA levels of TLR4/NF- $\kappa$ B in myocardial cells after CPR, with or without intravenous administration of SFI.

\section{METHODS}

\section{Preparation of Experimental Animals}

Wuzhishan miniature pigs (30 pigs) from Chinese Academy of Agricultural Sciences, male inbred, 12-14 months old, weighing $30 \pm 2 \mathrm{~kg}$, were randomly divided into a CPR group of $24 \mathrm{~h}$ and a sham operation group of $6 \mathrm{~h}$ using a random number table. Intramuscular injection of ketamine at $20 \mathrm{mg} / \mathrm{kg}$ combined with ear vein injection of propofol at $2 \mathrm{mg} / \mathrm{kg}$ was administered to induce anesthesia, with an initial dose of intravenous bolus injection of $0.25 \mathrm{mg}$ remifentanil, followed by continuous intravenous infusion at $30-50 \mu \mathrm{g} /(\mathrm{kg} \cdot \mathrm{h})$ for analgesia. During the anesthesia maintenance phase, an initial dose of intravenous bolus injection of $3 \%$ pentobarbital sodium was given at $30 \mathrm{mg} /(\mathrm{kg} \cdot \mathrm{h})$, followed by continuous intravenous infusion at $8 \mathrm{mg} /(\mathrm{kg} \cdot \mathrm{h})$. Orotracheal intubation was performed to mechanical ventilation, under the control of a $\mathrm{CO}_{2} \mathrm{SMO}$ plus respiratory monitor. Inhaled oxygen concentration was $21 \%$, with ventilation frequency of $12 \mathrm{times} / \mathrm{min}$. Tidal volume (VT) was initially set at $8 \mathrm{~mL} / \mathrm{kg}$, and the ventilator was adjusted to make the end tidal carbon dioxide $\left(\mathrm{PETCO}_{2}\right)$ maintained at $35-40 \mathrm{~mm} \mathrm{Hg}$. A $7 \mathrm{~F}$ angiography catheter was inserted into the right femoral vein through a sheath, and a bipolar temporary pacing electrode was placed to the right ventricle. A $5 \mathrm{~F}$ Outlook catheter was inserted into the aortic arch through the right femoral artery, to which a pressure transducer and a PICCO monitor were connected to measure the aortic pressure. Cardiac output was measured using thermal dilution method. ${ }^{(7)}$

\section{Procedures}

The endocardial electrical stimulation method was employed. Intraventricular lead was connected to medical program-controlled stimulator (GY-600A;
Kaifeng Huanan Equipment Co., Ltd., China), and esophageal output S1S2 (300/200 ms) model was chosen. Continuous electrical stimulus at $8: 1$ ratio and step size of $10 \mathrm{~ms}$ was commenced until occurrence of ventricular fibrillation (VF). The criteria of VF were a rapid drop of the arterial blood pressure and a $V F$ waveform in ECG. ${ }^{(9)}$ Eight minutes after VF, chest compressions was performed at a frequency of 100 times/min. CPR group was randomly divided into 3 groups according to a random number table, namely, a Shenfu (SFI) group, a epinephrine (EP) group and a saline (SA) group. Animals in 3 groups were given SFI $(1.0 \mathrm{~mL} / \mathrm{kg}), \mathrm{EP}(0.02 \mathrm{mg} / \mathrm{kg})$ and SA, respectively, which were prepared in $20 \mathrm{~mL}$ solutions and injected into the central vein. If no ROSC occured, defibrillation was performed at $120 \mathrm{~J}$, followed by continuous chest compressions for $2 \mathrm{~min}$. If there's still no spontaneous circulation, a second defibrillation was performed at $150 \mathrm{~J}$. There was a 6 -min interval following the 2-min CPR to analyze the rhythm of the heart, and another 2-min CPR was performed according to the requirements of each group and the next defibrillation was prepared. CPR quality was monitored by a HeartStart MRx Monitor/Defibrillator with Q-CPR, and operators were switched after every 2-min CPR to avoid fatigue that may affect the quality of CPR. The criteria of ROSC included systolic pressure remained $\geqslant 80 \mathrm{~mm} \mathrm{Hg}$ for at least $10 \mathrm{~min}$. If ROSC was not achieved after 30 min of resuscitation, failure of CPR and animal death were announced..$^{(7)}$ For the animals in the sham group $(n=6)$, the same procedure was performed, including anesthesia, mechanical respiration, and catheter indwelling, but without defibrillation and compression. Including heart rate $(\mathrm{HR})$, cardiac output $(\mathrm{CO})$, mean arterial pressure (MAP), were measured continuously, and the values at baseline and $30 \mathrm{~min}, 2,4$, and $6 \mathrm{~h}$ after ROSC were recorded.

\section{Histopathology}

Some myocardial specimens were preserved in $4 \%$ paraformaldehyde to observe pathologic changes for light microscope and transmission electron microscopy (TEM).

The slides were deparaffinized with xylene twice for 5-10 $\mathrm{min}$, followed by absolute ethanol for $5 \mathrm{~min}$, $90 \%$ ethanol for $2 \mathrm{~min}, 70 \%$ ethanol for $2 \mathrm{~min}$ and distilled water for $2 \mathrm{~min}$. Then, the slides were stained in hematoxylin solution for $15 \mathrm{~min}$, and flushed with tap water for $10 \mathrm{~min}$, then stained in eosin for $1 \mathrm{~min}$ 
(time can be adjusted according to the staining results and experimental requirements), and finally washed twice with $70 \%$ ethanol. Dehydration, clearing and mounting: The slides were dehydrated twice with 95\% ethanol for $2 \mathrm{~min}$, cleared twice with xylene for $5 \mathrm{~min}$, and then mounted with neutral balsam. Nuclei should be blue, while cytoplasm should be pink or red through microscopic observation.

Myocardial specimens were fixed with 1.5\%-3\% glutaraldehyde for 1-2 $\mathrm{h}$, then they were fixed with $1 \%$ starving acid for $1-2 \mathrm{~h}$. After that the specimeds were washed with $0.1 \mathrm{~mol} / \mathrm{L}$ PBS twice for 5-10 min, and were dehydrated by $50 \%, 70 \%, 90 \%$ ethanol each time (5-10 min/each time), respectively. The samples were then dehydrated by $100 \%$ ethanol for three times (5-10 $\mathrm{min} /$ time), and by propylene oxide for $10 \mathrm{~min}$ at room temperature. Following that, they were embedded by Epon812, and were polymerized at $40{ }^{\circ} \mathrm{C}$ for $2 \mathrm{~h}$, at $60{ }^{\circ} \mathrm{C}$ for $4 \mathrm{~h}$, at $80{ }^{\circ} \mathrm{C}$ for $10 \mathrm{~h}$, respectively. Semi thin section was first stained by toluidine blue for $1 \mathrm{~min}$, then stained by uranium acetate and lead citrate for 15 min each time, and finally observed under the electron microscopy $(\mathrm{H}-7650$; HITACHI, Tokyo, Japan).

\section{Enzyme Linked Immunosorbent Assay for Serum Cytokine Levels}

The $2 \mathrm{~mL}$ vein blood was centrifuged at $35,000 \times \mathrm{g}$ for 10 min at $4{ }^{\circ} \mathrm{C}$, centrifuged blood was collected and immediately frozen at $-80{ }^{\circ} \mathrm{C}$ for enzyme linked immunosorbent assay (ELISA, Sunbio Biotech Co. Ltd., China), Microtitration plates were coated overnight with $50 \mu \mathrm{L}$ of a $10 \mu \mathrm{g} / \mathrm{mL}$ solution of Biotin anti-TNF- $\alpha$ and IL-6 in carbonate buffer $\mathrm{pH}$ 9.6. After blocking and washing, the sample was incubated for $30 \mathrm{~min}$ at room temperature. Each well was added with $50 \mu \mathrm{L}$ color liquid and stop solution, gently mixed for $30 \mathrm{~s}$ and incubated for $15 \mathrm{~min}$ at room temperature. The absorbance at $450 \mathrm{~nm}$ was measured after 30 min using an ELISA plate reader. Cytokine concentrations were obtained from a standard curve. Duplicate readings were obtained for all samples and the means were calculated.

\section{Western Blot Analysis of TLR4 and NF- $\kappa$ B}

A 100-mg frozen heart sample was homogenized and then centrifuged at $12,000 \mathrm{r} / \mathrm{min}$ for $10 \mathrm{~min}$ at $4{ }^{\circ} \mathrm{C}$. A total of $100 \mu \mathrm{g}$ of protein was loaded onto $10 \%$ SDS-PAGE gel in each sample. Western blotting was performed with the membranes blocked for $2 \mathrm{~h}$ with
$5 \%$ non-fat milk and then incubated with the primary antibodies (diluted overnight at $4{ }^{\circ} \mathrm{C}$ ): TLR4 and NF- $\kappa$ B, 1:200 (AbcamBiotechnology, UK); and GAPDH, 1:250 (Santa Cruz Biotechnology, USA). Blots were blocked and incubated at $4{ }^{\circ} \mathrm{C}$ overnight with the specific primary antibody. The immunoreactive bands were visualized on film and scanned. The data were analyzed by Image Pro Plus (version 4.1, Media Cybernetics). The quantitative data from Western blot bands were expressed as the target protein OD/GAPDH OD ratio.

Reverse Transcriptase-PCR Assay for TLR4 and
NF- $\kappa$ B
Total RNA was extracted from 50 to $100 \mathrm{mg}$ of heart tissue according to the protocol described for the BioEasy SYBR Green I Real-Time PCR Kit Manual (Bo Ri Technology Co., Ltd., China). The primer sequences of the expected PCR products were as follows: for TLR4, Forward primer: 5'-GGGTCACTTCTGTTCACG-3' and reverse primer: 5'-GATGTTGTCAGG-GATTTT-3'; for NF- $\kappa$ B, forward primer: 5'-ATTTCGTTTCCGTTATGTG-3', and reverse primer: 5'-CTGAGGGTAGGACTTCTTG-3'. Preincubation was performed at $94{ }^{\circ} \mathrm{C}$ for $2 \mathrm{~min}$, followed by amplification at $94{ }^{\circ} \mathrm{C}$ for $30 \mathrm{~s}, 72{ }^{\circ} \mathrm{C}$ for $60 \mathrm{~s}$, and finally, during slow heating up, $72{ }^{\circ} \mathrm{C}$ for $10 \mathrm{~min}$. After the amplification, melting curve analysis with a temperature gradient from $65{ }^{\circ} \mathrm{C}$ to $94{ }^{\circ} \mathrm{C}$ was recorded every $0.5{ }^{\circ} \mathrm{C}$. Relative quantification is generally calculated with the $2^{-\Delta \Delta C T}$ formula by the comparative $\mathrm{Ct}$ method, the copy number of the target gene $2^{-\triangle \triangle C T}=2^{-(\triangle C T \text { target gene }-\triangle C \text { CT GAPDH gene })}$.

\section{Statistical Analysis}

The experimental data were analyzed by SPSS 17.0 (SPSS Inc, Chicago, III, USA). The results are expressed as mean \pm standard deviation $(\bar{x} \pm s)$, and Student's $t$ test was used for comparisons between two groups. Differences at different time points within groups were compared with repeated-measures analysis of variance (ANOVA). A two-tailed value of $P<0.05$ was considered significant.

\section{RESULTS}

\section{Modeling and Resuscitation Outcomes}

Six animals were successfully resuscitated with a ROSC rate of $83.3 \%$ in each of the 3 groups. CPR time to ROSC, defibrillation frequency and defibrillation energy in the EP and SFI groups were significantly different from that of the SA group, while 
no significant differences were found between the SFI group and the EP group in terms of the ROSC rate, CPR time to ROSC, defibrillation frequency and defibrillation energy (Table 1).

\section{Table 1. Resuscitation Outcome in} Swine among Groups $(\bar{x} \pm s)$

\begin{tabular}{lcccc}
\hline Group & $n$ & $\begin{array}{c}\text { Number of } \\
\text { shock }\end{array}$ & Energy of shock $(\mathrm{J})$ & $\begin{array}{c}\text { Time to ROSC } \\
(\mathrm{min})\end{array}$ \\
\hline SA & 6 & $5.51 \pm 2.52$ & $795.00 \pm 375.68$ & $10.00 \pm 3.79$ \\
EP & 6 & $2.75 \pm 1.66^{*}$ & $312.70 \pm 134.28^{* *}$ & $6.01 \pm 2.17^{* *}$ \\
SFI & 6 & $2.61 \pm 1.03^{*}$ & $332.51 \pm 168.39^{* *}$ & $5.00 \pm 1.69^{* *}$ \\
\hline
\end{tabular}

Notes: SA, saline; EP, epinephrine; SFI, Shenfu Injection; ROSC, restoration of spontaneous circulation. ${ }^{*} P<0.05,{ }^{* *} P<0.01$ vs. SA (one-way repeated-measures ANOVA)

\section{Hemodynamics}

As shown in Figure 1, differences in MAP and $\mathrm{CO}$ between the CPR group and the sham group were not statistically significant at the baseline $(P>0.05)$, and MAP in the SA group, however, was significantly higher than that of the EP and SFI groups after ROSC $(P<0.05)$. On the contrary, MAP in the SFI group was not different from that of the EP group at $30 \mathrm{~min}$ and $2 \mathrm{~h}$ after ROSC, respectively, and MAP in the SFI group was significantly increased compared with that of the EP group at $6 \mathrm{~h}$ after ROSC $(P<0.05)$. Similarly, $C O$ in the CPR group was significantly decreased compared with that of the sham group after ROSC $(P<0.01)$. CO in the SFI and EP groups was significantly higher than the SA group at $30 \mathrm{~min}$ and $2 \mathrm{~h}$ after ROSC $(P<0.05)$, and $C O$ of the $S F I$ group was significantly higher than the EP group at $6 \mathrm{~h}$ after ROSC $(P<0.05)$.

\section{Histopathology}

Myocardial cells of the sham group showed normal structure under light microscope (Figure 2A).
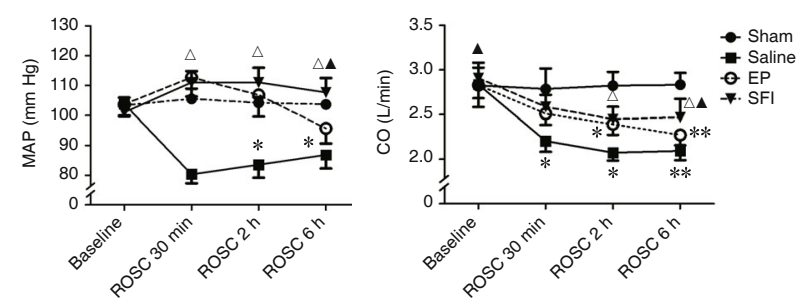

Figure 1. Comparison of Hemodynamics and Oxygen Metabolism among Groups $(n=6, \bar{x} \pm s)$

Notes: SFI, Shenfu Injection; ROSC, restoration of spontaneous circulation; MAP, mean aortic pressure; CO, cardiac output; $1 \mathrm{mmHg}=0.133 \mathrm{kPa} ;{ }^{*} P<0.05,{ }^{* *} P<0.01$ vs. sham (one-way repeated-measures ANOVA); ${ }^{\triangle} P<0.05$ vs. saline; ${ }^{\Delta} P<0.05$ vs. EP (student's $t$ test).

Massive swelling, necrosis of myocardial cells, and ruptured myocardial fibers were found in the CPR group (Figures 2B and 2C). Level of myocardial cell necrosis in the SFI group was significantly reduced (Figure 2D). While Funder electron microscopy, the sham group showed intact myocardial ultrastructure, neatly arranged myofilaments, clear Z- and M-line of sarcomere, normal nuclear structure, and normallyshaped mitochondria (Figure 2E). Mitochondria in the myocardial cells of the CPR group was swelling, with vacuolar degeneration and damaged cristae and outer membranes, and Z- and M-line of sarcomere was ruptured (Figure $2 F$ and $2 \mathrm{G}$ ). Destruction of myocardial cells in the SFI group was significantly reduced with partial nuclear chromatin condensation, and mitochondrial swelling and vacuolation was also significantly reduced. The Z- and M-line of sarcomere remained visible (Figure $2 \mathrm{H}$ ).

\section{ELISA Analysis of IL-6 and TNF- $\alpha$ Levels}

As shown in Figure 3, serum IL-6 and TNF- $\alpha$ levels were significantly increased throughout the study

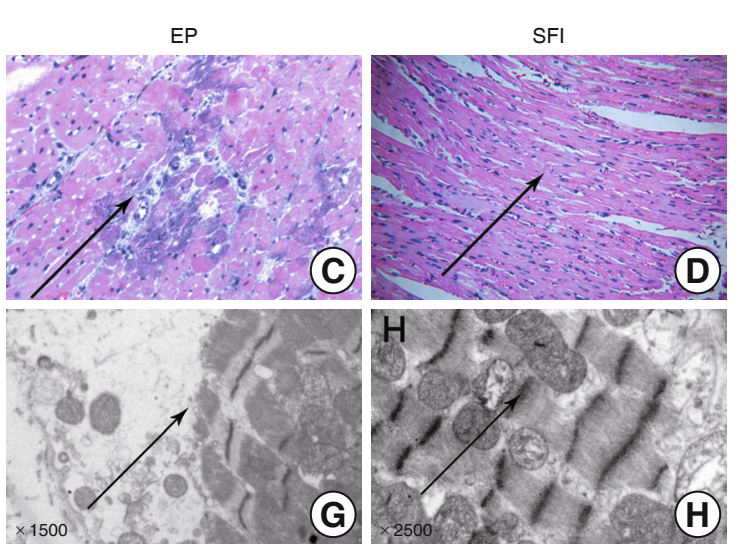

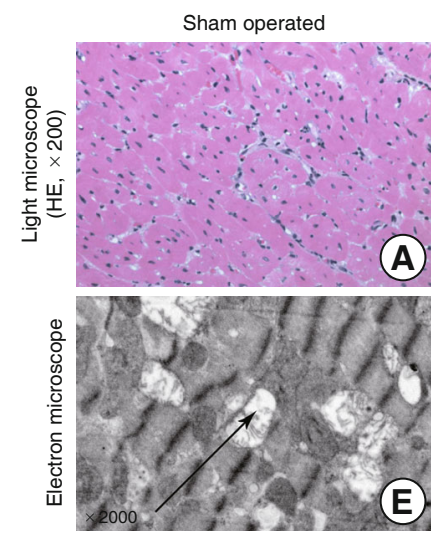

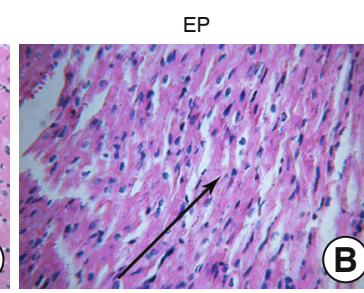

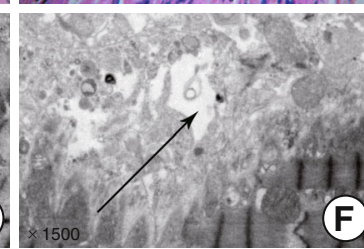

Figure 2. Cytoplasmic Ultrastructure and Histopathology Photographs of the Myocardium

Notes: (A) normal architecture of cardiomyocytes: (B, C, F and G) myofibril organelles were extensively damaged, and the myocardium exhibited progressive, severe deterioration (arrows): (E) normal mitochondrial architecture (arrows): (D and H) mitochondrial architecture of cardiomyocytes exhibited little intracellular damage in the SFI group at $24 \mathrm{~h}$ after cardiac resuscitation (arrows) 
time points after ROSC compared with baseline values in all three resuscitation groups $(P<0.05$ or $P<0.01)$. However, IL-6 and TNF- $\alpha$ were significantly decreased in the SFI group compared with the SA and EP group at $6 \mathrm{~h}(P<0.05$ or 0.01$)$; they were lower in the SFI group than in the SA and EP groups at $24 \mathrm{~h}$ after successful resuscitation $(P<0.05)$.

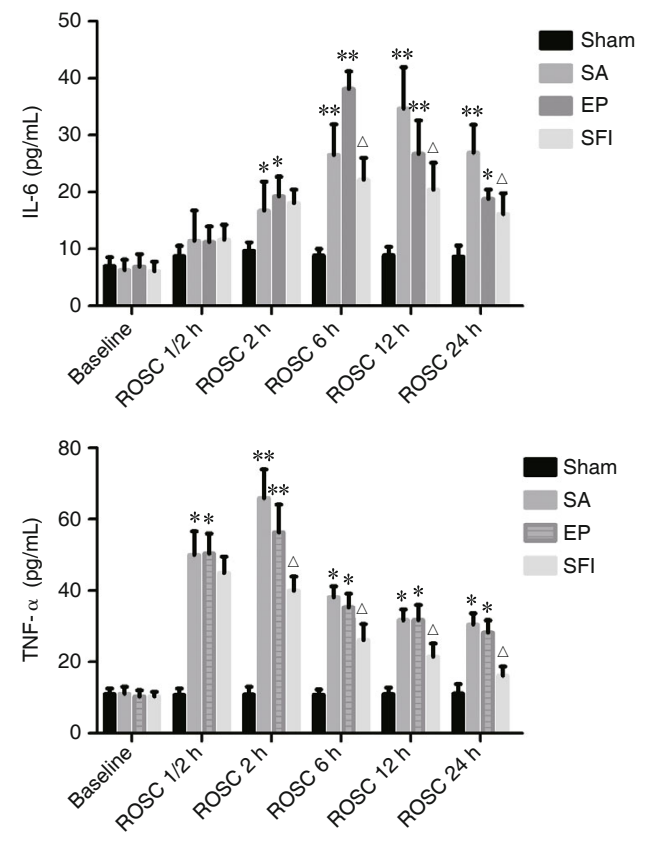

Figure 3. ELISA Analysis of IL-6, TNF- $\alpha$ Levels in Serum of Pig at $24 \mathrm{~h}$ after ROSC $(n=6, \bar{x} \pm s)$ group

Notes: ${ }^{*} P<0.05,{ }^{* *} P<0.01$ vs. sham group, ${ }^{\triangle} P<0.05$ vs. EP

\section{Protein Expressions of TLR4 and NF- $\kappa$ B}

As shown in Figure 4, Western blot analysis showed that the protein expressions of TLR4 and NF- $\kappa \mathrm{B}(P<0.05$ or 0.01$)$ progressively increased in the SA, EP and SFI groups compared with the shamoperated group. The levels of TLR4 and NF- $\kappa$ B were significantly decreased in the SFI group than in the EP group at $24 \mathrm{~h}$ after ROSC $(P<0.05)$.

\section{mRNA Levels of TLR4 and NF- $\kappa$ B}

The copy number of the TLR4 and NF- $\kappa$ B gene in the SA, EP, and SFI groups significantly increased compared with that in the sham-operated group $(P<0.05$ or $P<0.01)$. Furthermore, the mRNA expression of TLR4 and NF- $\kappa$ B in the SFI group was reduced compared with the EP and SA group at $24 \mathrm{~h}$ after ROSC $(P<0.05)$.

\section{DISCUSSION}

Post-resuscitation myocardial dysfunction is

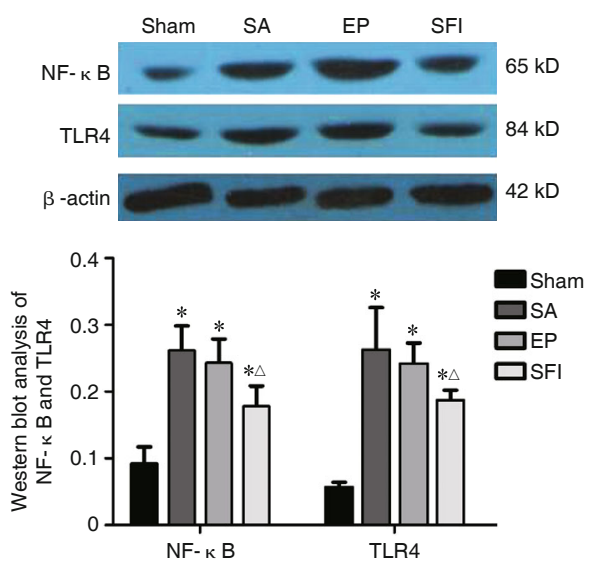

Figure 4. Quantification of TLR4 and NF- $\kappa$ B Protein Levels in Myocardial Tissue of Pig in Groups at $24 \mathrm{~h}$ after $\operatorname{ROSC}(n=6, \bar{x} \pm s)$ Notes: ${ }^{*} P<0.01$ vs. sham group, ${ }^{\triangle} P<0.05$ vs. EP group

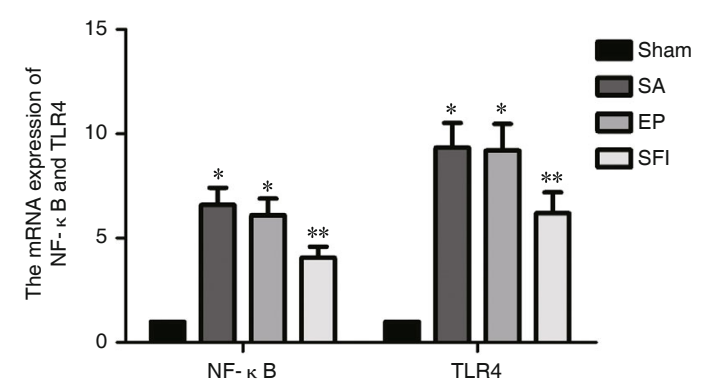

Figure 5. mRNA Expressions of TLR4 and NF- $\kappa$ B in Myocardial Tissue of Pig by RT-PCR $(n=6, \bar{x} \pm s)$ Notes: ${ }^{*} P<0.01$ vs. sham group, ${ }^{\triangle} P<0.05$ vs. EP group

one of the main causes of death in patients with CA. Coronary artery blood flow was decreased or interrupted after the occurrence of $\mathrm{CA}$, and global ischemia of the heart induces myocardial stunning. Early after ROSC, ischemia/reperfusion injury occurs in myocardial cells and hemodynamic disturbance is aggravated, leading to cardiogenic shock, accompanied by systemic low blood volume and vasomotor dysfunction. ${ }^{(10)}$ Meanwhile, CA represents the most severe form of shock, during which oxygenated metabolites cannot be eliminated and a large amount of lactic acid deposits in myocardial cells, directly leading to myocardial damage. The underlying mechanism is similar to that of myocardial damage induced by septic shock. ${ }^{(11)}$ The present study found that severe myocardial dysfunction occurred in miniature pigs within $6 \mathrm{~h}$ after ROSC, which was manifested in the considerably decreased MAP and $\mathrm{CO}$, as well as serious damage of myocardial cell ultrastructure.

Unstable hemodynamics and microcirculatory disturbance due to post-resuscitation myocardial 
dysfunction will cause persisted oxygen debt and subsequent activation of endothelial cells and release of inflammatory factors (such as TNF- $\alpha$ ), leading to systemic inflammatory response. ${ }^{(11)}$ TNF- $\alpha$, one of the early proinflammatory cytokines, is a key cytokine involved in ischemia-reperfusion injury. ${ }^{(12)}$ It induces the massive expression of various inflammatory mediators, such as TNF- $\alpha$ and IL-6, by activating neutrophils, and results in "cascade reactions", which further amplify TNF- $\alpha$ expression in a positive feedback manner, and TNF- $\alpha$, in turn, activates $\mathrm{NF}-\kappa \mathrm{B}$. This fuels a vicious circle of uncontrolled inflammatory cytokine release, which directly impair the myocardial cells, resulting in myocardial dysfunction. ${ }^{(6)}$ In the present study, we found that the serum levels of TNF- $\alpha$ and IL- 6 in miniature pigs in the CPR group were significantly increased at $24 \mathrm{~h}$ after ROSC, which was similar to the mechanism of myocardial dysfunction secondary to sepsis.

NF- $\kappa$ B is a dimer composed of two subunits, $\mathrm{P} 50$ and P65. Under normal conditions, it is bound by the inhibitor of NF- $\kappa$ B (I- $\kappa$ B) to form an inactive trimer and is sequestered in the cytoplasm. When the body is stressed by infection, shock, and reperfusion, $\mathrm{NF}-\kappa \mathrm{B}$ dissociates from $\mathrm{I}-\kappa \mathrm{B}$ and translocates into nucleus, where it binds to specific promoters and regulate gene expression, and may lead to uncontrolled systemic inflammatory response and cell apoptosis. ${ }^{(13)}$ Activated NF- $\kappa \mathrm{B}$ can initiate and regulate various proinflammatory cytokines and cellular adhesion molecules, causing neutrophil adhesion and aggregation as well as cascade amplification of cellular inflammatory response. ${ }^{(14)}$ TLR4, an important member of the TLR family, can specifically recognize LPS on the cell walls of gram negative bacteria and is considered as the major LPS receptor. ${ }^{(6)}$ In the early stage after ROSC, the body experiences ischemia/reperfusion process and produces a large amount of metabolites such as free oxygen radicals and lactic acid, leading to systemic inflammatory responses and hypoimmunity. Therefore, the body is often associated with severe bacterial (gram negative bacteria) infection, which causes the release of LPS and intestinal translocation of LPS. ${ }^{(15)}$ LPS binds to receptor CD14 on monocyte/ macrophage membrane, and then to receptor TLR4 to transfer the signal into the cell. When TLR4 is activated, it interacts with NF-kB to produce large amounts of oxygen free radicals and releases massive inflammatory mediators. ${ }^{(16)}$ Thereby the downstream TNF- $\alpha$ and other inflammatory factors are activated, directly leading to myocardial cell injury. Studies have shown that TLR4 is involved in myocardial dysfunction in ischemia/reperfusion. ${ }^{(17)}$ It was found in the present study that the myocardial function of pigs was seriously impaired after ROSC, and the protein and mRNA levels of TLR4/NF-kB in myocardial cell of the CPR group were significantly higher than that of the sham group at $24 \mathrm{~h}$ after ROSC.

SFI is derived from a modified classic prescription of Chinese medicine "Shenfu Decoction (参附汤)" and the active ingredients include ginsenosides and n-desmethyl aconitine. Ginsenoside can also activate ribonuclease, which increases the biosynthesis of DNA and promotes the metabolism of sugar, protein and fat. This helps myocardial repair and myocardial cell energy supply, increases myocardial contractility, and improves myocardial function. ${ }^{(18)}$ In addition, ginsenosides also prevent against stress, oxidation and ischemia and hypoxia. They may also eliminate free-radicals, prevent lipid peroxidation, reduce intracellular calcium overload, and stabilize the structure of cell membrane. ${ }^{(19)}$ Studies have shown that SFI can protect the brain tissue by scavenging free oxygen radicals, inhibiting the inflammatory response, and reducing cell edema in ischemia-reperfusion injury. ${ }^{(20)}$ In addition, Qiu Z, et al ${ }^{(21)}$ suggested that SFI could modulate severe immune disturbances, maintain a proinflammatory/anti-inflammatory balance, and improve clinical outcomes. In our previous studies, SFI was found to inhibit inflammatory responses, regulate Th1/Th2 cell imbalances, and improve postresuscitation myocardial immune dysfunction. ${ }^{(22)}$ In addition, we also observed that SFI could improve immunity and inhibit inflammatory responses by improving the apoptosis of splenic T lymphocytes. ${ }^{(23)}$ In the study by Wang, et $\mathrm{al}^{(24)}$ rats with endotoxininduced systemic inflammatory response syndrome were treated with $\mathrm{SFI}$, and the results showed that SFI significantly reduced the activity of NF- $\kappa$ B and the levels of IL- 6 and TNF- $\alpha$, and alleviated the pathological damage of lung and liver.

We found in the present study that both serum IL- 6 and TNF- $\alpha$ levels and TLR4/NF- $\kappa$ B expression in myocardial cells in the SFI group were significantly reduced, suggesting that SFI may reduce the activity of NF- $\kappa$ B and TNF- $\alpha$ levels by inhibiting the 
inflammatory response, so as to block the inflammatory response and cell apoptosis regulated by NF- $\kappa \mathrm{B}$, and to reduce myocardial cell injury. This may serve as one of the main mechanisms underlying the preventive and therapeutic effects of SFI for post-resuscitation myocardial dysfunction. In addition, our results also suggested that SFI may enhance the balance of SIRS by inhibiting the overexpression of proinflammatory mediators, thereby reducing systemic inflammatory response and regulating immune function.

\section{Conflict of Interest}

The authors declare that they have no conflicts of interest.

\section{Author Contributions}

Gu W and Li CS conceived and designed the experiments. Gu W, Zheng PY and Hou XM performed the experiments. Gu W and Hou XM analyzed the data. Hou XM contributed reagents/materials/analysis tools. Gu W and Li CS wrote the paper.

\section{REFERENCES}

1. Chang $\mathrm{WT}$, Ma MH, Chien $\mathrm{KL}$, Huang $\mathrm{CH}$, Tsai MS, Shih FY, et al. Postresuscitation myocardial dysfunction: correlated factors and prognostic implications. Intensive Care Med 2007;33:88-95.

2. Chung SP, Song FQ, Yu T, Weng Y, Sun S, Weil MH, et al. Effect of therapeutic hypothermia vs delta-opioid receptor agonist on post resuscitation myocardialfunction in a rat model of CPR. Resuscitation 2011;82:350-354.

3. Gu W, Li CS, Yin WP, Guo ZJ, Hou XM, Zhang D. Apoptosis is involved in the mechanism of postresuscitation myocardial dysfunction in a porcine model of cardiac arrest. Am J Emerg Med 2012;30:2039-2045.

4. Huang $\mathrm{Y}, \mathrm{He} \mathrm{Q}$. The relationship between sarcplasmic reticulum $\mathrm{Ca}^{2+}$ modulation proteins and postresuscitation myocardial dysfunction. Chin Critic Care Med (Chin) 2014;26:697-700.

5. Gu W, Li CS, Yin WP, Hou XM, Zhang J, Zhang D, et al. Expression imbalance of transcription factors GATA-3 and T-bet in post-resuscitation myocardial immune dysfunction in a porcine model of cardiac arrest. Resuscitation 2013;84:848-853.

6. Chen J, Jiang H. TLR4 signal pathway and inflammation reaction. Med Recapitulate 2009;15:2902-2904.

7. Gu W, Li C, Yin W, Guo Z, Hou X, Zhang D. Shenfu Injection reduces postresuscitation myocardial dysfunction in a porcine model of cardiac arrest by modulating apoptosis. Shock 2012;38:301-306.

8. Ji XF, Yang L, Zhang MY, Li CS, Wang S, Cong LH. Shenfu Injection attenuates postresusbitation myocardial dysfunction in a porcine model of cardiac arrest. Shock 2011;35:530-536.

9. Gu W, Li C, Yin W, Guo Z, Hou X, Zhang D. Effects of different resuscitation modalities on post-resusciatation myocardial apoptosis in porcine models of cardiac arrest. Chin J Emerg Med (Chin) 2013;22(3):8-13.
10. Jones AE, Shapiro NI, Kilgannon JH, Trzeciak S. Goaldirected hemodynamic optimization in the post-cardiac arrest syndrome: a systematic review. Resuscitation 2008;77:26-29.

11. Ma G, Hong GL, Zhao GJ, Li MF, Wu B. Changes and significance of plasma $B$-type natriuretic peptide and cardiac troponin I in patients with sepsis. Chin J Tradit Chin West Med Crit Care (Chin) 2014;21:99-103.

12. Liu ZJ, Liu XL, Zhao J, Shi YJ, Yan LN, Chen XF, et al. The effects of SOCS-1 on liver endotoxin tolerance development induced by a low dose of lipopolysaccharide are related to dampen NF-kappaB-mediated pathway. Dig Liver Dis 2008;40:568-577.

13. Varfolomeev EE, Ashkenazi A. Tumor necrosis factor: an apoptosis JuNKie? Cell 2004;116:491-497.

14. Sun B, Fan H, Honda T, Fujimaki R, Lafond-Walker A, Masui $Y$, et al. Activation of NF kappa $B$ and expression of ICAM-1 in ischemic-reperfused canine myocardium. $\mathrm{J} \mathrm{Mol}$ Cell Cardiol 2001;33:109-119.

15. Gu W, Li C, Yin W, Guo Z, Hou X, Zhang D, et al. Expression imbalance of transcription factors GATA-3 and T-bet is involved in the mechanism of postresuscitation lung injury. Chin J Emerg Med 2014;23:8-14.

16. $\mathrm{Xu} \mathrm{K,} \mathrm{Gu} \mathrm{W,} \mathrm{Hou} \mathrm{XM.} \mathrm{Role} \mathrm{of} \mathrm{TLR4/NF-} \kappa$ B signal pathway in the pathogensis of acute myocardial dysfunction after resuscitation. Chin Critic Care Med (Chin) 2015;27:190-196.

17. Cha J, Wang Z, Ao L, Zou N, Dinarello CA, Banerjee A, et al. Cytokines link Toll-like receptor 4 signaling to cardiac dysfunction after global myocardial ischemia. Ann Thorac Surg 2008;85:1678-1685.

18. Zhang Y, Li CS, Wu CJ. Neuroprotective effect of Shenfu Injection following cardiac arrest in pig correlates with improved mitochondrialfunction and cerebral glucose uptake. Chin J Integr Med 2014;20:835-43.

19. Zhang XJ, Song L, Zhou ZG, Wang XM. Effect of Shenfu Injection on gastrointestinal microcirculation in rabbits after myocardial ischemia-reperfusion injury. World $\mathrm{J}$ Gastroenterol 2006;12:4389-4391.

20. Hou X, Li C, Gu W, Guo Z, Yin W, Zhang D. Effect of Shenfu on inflammatory cytokine release and brain edema after prolonged cardiac arrest in the swine. Am J Emerg Med 2013;31:1159-1164.

21. Ze LQ, Yi PY, Ning Z. Clinical efficacy of Shenfu Injection in treating severe sepsis and its effects on serum levels of interleukin-6 and interleukin-10. Chin Integr Tradit West Med Intens Critic (Chin) 2012;32:348-351.

22. Gu W, Li C, Yin W, Hou X. Effects of Shenfu Injection on the expression of transcription factors T-bet/GATA-3 in pigs with post-resuscitation myocardial dysfunction. Chin Critic Care Med 2015;27:190-196.

23. Gu W, Zhang Q, Li CS. Effects of Shenfu Injection on apoptosis of regulatory $T$ lymphocytes in spleen during post-resuscitationimmune dysfunction ina porcine model of cardiac arrest. Chin J Integr Med 2016;22:666-73.

24. Wang J, Qiao LF, Yang GT. Role of Shenfu Injection in rats with systemic, inflammatory response syndrome. Chin J Integr Med 2008;14:51-55.

25. Zheng CD, Min S. Cardioprotection of Shenfu Injection against myocardial ischemia/reperfusion injury in open heart surgery. Chin J Integr Med 2008;14:10-16.

(Accepted May 29, 2018; First Online January 9, 2021) Edited by ZHANG Wen 